\title{
EVIDENCE-BASED STROKE REHABILITATION
}

\author{
Ivona D. Stankovic ${ }^{1,2}$, Anita Stankovic ${ }^{1}$, Marija Spalevic ${ }^{I}$, Dragan Zlatanovic ${ }^{1}$, Tamara Stankovic ${ }^{1}$ \\ ${ }^{1}$ Clinic for Physical Therapy and Rehabilitation, Clinical Center Niš, Serbia \\ ${ }^{2}$ Faculty of Medicine, University of Niš, Serbia
}

\begin{abstract}
Stroke can have different clinical characteristics and consequences, with unequal disability and outcome, thus demanding individual approach, specific skills and general knowledge. Treatment of stroke has significantly improved during the last twenty years, mainly because of clinical and experimental studies, adequate medicamentous therapy, and the use of new technologies as well. Use-dependent rehabilitation strategy includes repetitive training with proper adjustment of the program. Other rehabilitation practices should also be incorporated, such as self-care, recreation, and home-based activities. Motivation of the patients, improving quality of life, functional independence, activities of daily life are crucial. Holistic approach means that the patient as a whole should be considered and treated. Scientific evidence is sufficient to confirm the necessity of physical rehabilitation of patients after stroke in order to achieve the optimal results. Current evidence on the effect of physical therapy in stroke rehabilitation is presented.
\end{abstract}

Key words: stroke, physical therapy, rehabilitation, evidence.

\section{Introduction}

Stroke represents non-traumatic injury of the brain, induced by occlusion or rupture of cerebral vascular supply causing neurologic deficit, mainly damage of motor control, sensation, cognition, disequilibrium, and sometimes leading to coma. Proposed modern term instead of stroke is cerebrovascular accident $[1,2]$.

Stroke is the fourth leading cause of death after heart failure, cancer, and lower respiratory tract diseses. Cerebrovacular disease is the most important cause of long term disability. Stroke mainly affects older individuals, with about $70 \%$ of stroke occurring in persons over 65 years, but it can also occur in children [1,2].

Different vascular pathologic factors cause stroke. Ischemic brain damage, whether thrombotic or embolic, predominates with approximately $85 \%$, while intracranial hemorrhage represents about $15 \%$ of all strokes. Clinical stroke syndromes and consequent impairment and disability are based on affected artery.

Predominant causes of stroke are: atherosclerosis, hypertension, smoking, hypercholesterolemia, diabetes mellitus, and other factors. Non-modifiable risk factors are age, sex, previous stroke, and race. Sedentary way of life predisposes to cardiovascular disease and stroke. When stroke affects the left hemisphere language, comprehension and communication can be seriously dam-

*Correspondence to: Prof. Ivona D. Stanković, MD, PhD

Faculty of Medicine, University of Niš,

81 Dr. Zoran Đinđić Blvd, 18000 Niš, Serbia

Phone: +381184226644

E-mail: ivona@medfak.ni.ac.rs

Received October $3^{\text {rd }}, 2016$, accepted for publication January $13^{\text {th }}, 2017$ aged, while stroke in the right hemisphere alters intuition, solving, reasoning, judgment and vision. Cognitive problems after stroke are often inadequately addressed, though they are the most important predictor of professional inability. Older patients with stroke often have bigger motor and cognitive affection due to reduced brain vessel density and ischemia [1-3].

Certain gender differences were noted concerning outcome of stroke. Women exhibit greater disability and have a lower rate of full recovery than men. However, age and psychosocial adjusment tend to be more influential than gender itself.

The first six hours and emergency medical management in acute stroke unit are crucial for treatment of stroke. This is followed by further diagnostic evaluation, stroke prevention, and rehabilitation [4,5].

\section{Rehabilitation of Stroke}

Neuroplasticity is defined as the ability of central nervous system to remodel after injury. Indeed, adaptive plasticity of adult brain after damage was confirmed. Both basic and clinical studies have confirmed that rehabilitation must accompany any other medical or surgical intervention in treatment of stroke.

Functional recovery after stroke can be improved using different sensorimotor techniques, such as: strengthening, balance training, postural control, and improving range of motion. Brunnstrom early movement technique, Bobath neurodevelopmental technique, proprioceptive neural facilitation, and task-oriented approach have traditionally been used, but without suffi- 
cient evidence and proof of better results of one technique over the other [6-8].

Upper limb rehabilitation after stroke can produce significant improvement, with adequate compensatory strategies and promotion of recovery of the limb. Constraint-induced movement therapy (CIMT) is based on the fact that patients with upper extremity loss are more dependent on unaffected arm, with negative feedback. Repetitive movements, reacquisition of skills and cortical reorganization are included in CIMT. The original and modified CIMT were investigated in clinical trials that showed improvement of motor control and functional skills. EMG triggered neuromuscular electrical stimulation (NMES) is adequate for stroke rehabilitation, since some clinical studies have confirmed improvement of motor function and better activity of affected hand [9].

Lower limb rehabilitation after stroke was primarily performed using body weight-supported treadmill training (BWSTT). The aim is to make step movement of the limb and to ameliorate bipedal walking. Many repetitions that are needed, increased weight bearing and action of two therapists pose limitations to this technique. Also, clinical studies have confirmed that BWSTT is also beneficial for cardiovascular fitness of stroke patients.

New technologies, such as robotic-assisted training, were used and studied for rehabilitation of upper and lower extremities after stroke. The advantages are: induced passive or assisted movements, tactile feedback and skill acquisition and easy repetition of movements. Further studies on robotic-assisted training are needed, particularly for rehabilitation of finger movements after stroke. Transcranial magnetic stimulation, cortical brain stimulation, and neuronal transplantation were applied in clinical studies, and autologous marrow stromal cells in preclinical trials were used, all with good results [10].

Spasticity increases motor impairment and disability after stroke through an increase of tonic stretch reflexes. Treatment of spasticity includes exercises and botulinum toxin injections. Daily exercises, application of static resting splints prevents contractures. Botulinum toxin is particularly effective and repeatable.

Cognition, language, and communication difficulties after stroke are frequent. Certain spontaneous recovery after stroke is expected, while aphasia tends to recover much slower. Rehabilitation is focused to improving speaking, understanding, reading, and writing, as well as developing compensatory techniques. Many treatment options exist for rehabilitation of aphasia [11].

Dysphagia after stroke is treated using compensatory mechanisms. Percutaneous gastrostomy has been used successfully in store patients with severe dysphagia.

Pain syndromes, and particularly shoulder pain, are frequent complains after stroke. Neuromuscular electrical stimulation (NMES) of affected muscles reduced pain, and improved activity of daily life.
Control of bladder and bowel can be altered after stroke. The treatment includes timed voiding, and adequate medication based on urodynamic tests, or even intermittent catheterization in some cases.

Psychosocial changes after stroke include: sadness, anxiety, depression, anger, frustration, confusion, and other. Coping with these problems is essential in rehabilitation program, so motivation, counseling, and support are used [12,13].

Medical comorbidities in stroke are numerous, frequent, and potentially devastating. Venous thromboembolism, pneumonia, hypertension, heart failure, diabetes mellitus, malnutrition, and psychological deconditioning are the most important and should be treated adequately [14].

\section{Organization and Levels of Rehabilitation}

Instead of classical treatment programs in inpatient and outpatient ward, recent stroke rehabilitation is organized as a continuum according to current patients demands that change over time. This resulted in significant improvement of overall results of rehabilitation. Healthcare system, community resources, and patient's medical condition and motivation are important for the optimal result $[15,16]$.

Early stroke rehabilitation prevents prolonged bed rest and complications. Exercises in bed, gait training, activities of daily life are executed. Early rehabilitation was more influential on the outcome than the duration of rehabilitation, as found in meta-analyses.

Acute hospital rehabilitation is a team program including doctors, nurses, and equipment. It is essential that a patient can tolerate treatment for three or more hours per day.

Long-term acute rehabilitation is indicated for persons with prolonged complex disease. Adequate respiratory care, nutrition, and medical treatment are applied as long as needed. The intensity of treatment and specific demands are organized according to the tolerance of the patients.

Outpatient day rehabilitation includes the same rehabilitation program without hospital stay. Medical stability and social support are mandatory.

Outpatient rehabilitation is centered to single-modality training provided by physical, occupational and speech-language therapist as well.

Home therapy is the most familiar and appropriate environment for the patient and physical therapy. Stability of medical condition and sufficient social support enable home rehabilitation.

Specialized equipment, such as: adapted feeding devices, bathing equipment, dressing and walking devices assist patients to become more independent and have better performance. They are included depending on the level of functional activity $[17,18]$. 


\section{Outcome of Rehabilitation}

Data from medical references on the effect of physical rehabilitation after stroke are numerous, but they are difficult to interpret adequately, because of used methodology, samples, different rehabilitation programs, and outcome measures. Outcome can be measured by: morbidity, mortality, impairment, hospitalization, functional consequences, and quality of life. The insufficient number of randomized studies for rehabilitation of different diseases was mentioned in the Report of Disability published by the World Health organization [19].

Analysis of patient's condition and improvement in post-stroke rehabilitation should be presented longitudinally in order to better document and compare the results of treatment. Such longitudinal follow up was found in only one third of the studies in the literature, while others are mainly cross sectional studies, often without mentioning the dynamics of changes. All the studies emphasize that physical therapy after stroke results in improvement of physical performance, functional status, and quality of life. Independence can be obtained by most of the patients, ranging from $65 \%$ to $85 \%$, with less favorable social and professional results, ranging from $45 \%$ to $65 \%$. The most prominent aspects with improvement are: locomotion, mobility, self-care, and sphincter control. Communication and social cognition are improved to a smaller rate $[19,20]$.

Predictors of outcome are numerous, and include: severity and type of impairment, cognitive factors, comorbidity, social support, and type and quality of rehabilitation. The list of influential factors is long: age, education, type, severity, and location of stroke, previous stroke, heart failure and other comorbidities, dementia, presence of coma, cognition, language, balance, bowel and bladder function, depression, motivation, support, and others. However, it is difficult to apply numerous data on prediction factors to each individual patient with stroke. Documentation of certain factors that are present is very important, but clinical implications are complex. Also, stoke can impede different aspects of physical, cognitive, emotional, and vocational functions.

Control group, without therapy, for the studies of stroke rehabilitation is mandatory for randomized controlled trials, but this is impossible and unethical. On the other side, many other factors (motivation, support, adaptability, etc.) can influence the effect of rehabilitation. Individuality and diversity of factors makes them difficult to compare and divide into clinical groups. Spontaneous recovery is also uneasy to differentiate from rehabilitation results. Variability in stroke recovery is a well-known fact. It depends on initial severity of hemiparesis, age, location and site of infarct, stroke type, nonmotor parameters, and impairment in the first 72 hours $[21,22]$.

The first randomized controlled trial of stroke rehabilitation was published in 1980. Ever since that, the studies have been documenting the improvement achieved by physical therapy of stroke. Statistically significantly better functional independence and outcome were universally reported. Treatment in stroke rehabilitation unit produced less mortality, earlier discharge and better functional scores.

Meta-analyses confirmed that focused stroke rehabilitation is significantly better than other groups, particularly for mobility, personal care, and perception. Also, home based physical therapy was found effective to improve the outcome. The most important contribution of all the studies performed on stroke rehabilitation is that they emphasize certain clinically important aspects for proper rehabilitation programs. They have confirmed the importance of focused personal care, mobility training, team work, and education of patients and families [22,23].

More than 1000 randomized controlled trials have been published concerning stroke rehabilitation. The best period for the studies is the first days after stroke, since this is when spontaneous biological recovery happens. However, most of the studies are conducted more than 6 months after the stroke. Better standardization of terms used for recovery, the differences between the experimental and control arms, precise definition of usual and standard care are needed to improve the reliability of conducted studies of post stroke rehabilitation [24-27].

Randomised controlled trials indicate that early supported discharge (EDS) after stroke with continued rehabilitation program at home gives good results, primarily in reduced dependency. At home, patients are instructed to problem solving skills, supported in daily activities, and assisted in achieving the previous activities. This is realized through professional visits of physiotherapists, and other included professionals of the team. EDS demands adequate organizational changes, defining desired performance to be achieved. Increased capacity to solve problems improves patients' selfefficacy and independence. Studies have also confirmed cost reduction with implementation of EDS [28].

Management of medical demands, change of behavior of life, treatment of emotional consequences of stroke are the mainstay of support and self-management of patients. Solving problems, making adequate decisions, use of resources, proper relationship with the medical team, and feedback are dominant aspects of post-stroke support.

Multidisciplinary health care team in recovery after stroke has now significantly improved the treatment and outcome of stroke patients. Stroke unit care and thrombolysis have reduced acute morbidity and mortality, but long term consequences of stroke are frequent, and deserve individual, permanent and team approach. Interdisciplinary teamwork adds the group effort and responsibility to each individual task in planning and treatment of stroke. Hospital stroke team consists of neurologists, physiotherapists, occupational therapists, speech and language therapists, nurses, and others. Cochrane review indicates that such team approach improves the results of treatment of stroke. Physical therapy of stroke effectively improves function and mobility, however, it is not determined which ap- 
proach gives the best result. Early supported discharge reduces the length of stay, and according to systematic Cochrane review adds to activities of daily life of stroke patients. Long term rehabilitation support of patients with stroke is essential for further improvement of outcome, independence, and active life [29].

Physical exercise was applied in stroke patients aiming to document physical dysfunctions, improve motor performance, and prevent further damage. References prove the importance of physical training for physical independence, quality of life, and reduction of cardiovascular complications.

Physical training improves cerebral angiogenesis, vasomotor reactivity, reduces apoptosis and activates motor unit. Also, pre-stroke physical activity can improve recovery with decreased brain damage and better motor outcome. It also reduces the initial stroke severity, edema, infarct volume, inflammation, metabolic disorders. Preventive effects of pre-stroke physical activity (both walking $1 \mathrm{~h} /$ day $/ 5$ day per week, or vigorous aerobic activity) are documented by better functional outcome, milder severity, and better motor improvement.

Both animal and human researches have documented positive effect of acute exercise on muscle activation regulation in cerebral ischemia. Decrease of motoneuronal recruitment from spinal and supraspinal tracts, and increase of cortical activation of the unaffected side, with the decrease on the affected one, confirm compensatory neural process.

Chronic exercise after stroke was more extensively studied. Literature clearly states that physical therapy is the first line intervention for reduction of chronic disability after stroke. Treadmill training in early phases after stroke induces neuroplasticity through influence on brain vasomotor function, angiogenesis, neurotrophic factors, apoptosis and inflammation. Adequate brain oxygenation is essential for decreasing volume of cerebral infarct, and for less functional damage. Regeneration of striatonigral and corticonigral projections in ischemic brain, increased mitochondrial biogenesis, and improved cholinergic homeostasis were found after intensive treadmill training in stroke. All these processes lead to reduced muscle fatigue, improved walking speed and endurance. Overall better sensorimotor functions are directly correlated to the increase of aerobic capacity.

Volume, intensity, frequency, and timing of training directly affect proper neuroplasticity and recovery. Early intense training in the first 24 hours is not recommended. More intense exercises after stroke led to better recovery, but the influence of intensity of training on neuroplasticity in stroke is insufficiently studied [30-34].

The combination of exercise and medicaments is essential for functional outcome after stroke. Indomethacin, D-amphetamine, S-nitorogluthatione, and others exhibited neurovascular protective effect after cerebral ischemia in preclinical trials. Clinical significance of these medicaments combined with training is to be confirmed in clinical studies.
Published studies confirm that aerobic training improves cardio respiratory fitness, functional recovery, endurance, and quality of life after stroke. Also, aerobic training reduced cognitive dysfunction, promoting neuroplasticity in post-stroke period. Such increased brain plasticity produces better executive functions and long term memory. New brain imaging technologies have documented the increase of both white and gray matter, better synaptic connections, and brain activation patterns after aerobic training. Functional Magnetic Resonance Imaging (fMRI) confirmed increased activation of brain regions for attention control and inhibitory functioning, while activation was reduced in behavior conflict regions. Neuroplasticity improved by aerobic training was further documented using measurement of circulating neurotrophic factors (brain derived neurotrophic factor, insulin-like growth factor-1, and vascular endothelial growth factor). However, the exact influence of circulating growth factors on cognition in older persons is now unclear $[35,36]$.

Only three clinical studies concerning the influence of aerobic training on cognitive functions after stroke were published. Two out of three studies found amelioration of cognitive dysfunction with better functional results, motor learning, and speed of information processing. On the other side, eight weeks after training, the long-term preservation of achieved results was absent. Patients with stroke, opposite to healthy persons, did not exhibit improvement of cognitive functions.

Aerobic training combined with other exercises in rehabilitation of stroke was also studied. The addition of muscle strengthening techniques of lower extremities improved attention, executive functions and voluntary motor control. Combination of aerobic exercises with stretching, balance and task-specific practice resulted in better verbal memory and flexibility of cognition, without influence on executive functions. Aerobic and behavioral training improved cognition, but without total recovery [35,36].

Few combinations of aerobic training and pharmacological treatment (memantine, citicoline, recombinant tissue plasminogen activator, S-nitrosoglutathione) improved motor recovery after stroke, but for cognitive function the results were less efficient. Promotion of cognition was more efficient when low-intensity aerobic exercises, starting one week after stroke, were applied.

Cognitive and motor functions in stroke patients must not be considered independently. Complex cognitive functions and sensorimotor control show complex interaction in the process of learning and control of specific movements. This fact is even more important for older persons, since functional brain reactivation makes motor and cognitive functions more interrelated.

Aerobic capacity is best ameliorated using moderate continuous aerobic training, with several 30-60 minutes of exercise sessions per week, and $40-80 \%$ of the maximal heart rate reserve. Training with higher intensity could increase aerobic fitness, and oxygen uptake. Muscular and cardiovascular adaptations were improved 
when high intensity interval exercise (repeated intense training with active or passive rest) was administered. Forced treadmill exercises were proven superior to other types of training. Rehabilitation program in subacute post stroke period also reduced the complications of prolonged inactivity.

So, current recommendations for application of aerobic exercises are based on the effect on cardiovascular and motor functions, and not on cognitive functions. Thus, there is no standard recommendation for exercises concerning recovery of cognitive functions after stroke [37,38].

Self-management support after stroke is important for reducing chronic disabilities. It includes all activities one has to perform in order to efficiently manage the long-term management of stroke effect on the person and his family. Meta-review of literature has confirmed the important impact of stroke on self-image, and different support needed in the whole period of poststroke rehabilitation and recovery. The following recommendations were made according to these facts: the support for stroke patients need to be changed according to the stage of recovery; collaboration of health care professionals enable adequate decisions of patients in different stages of stroke recovery; individual psychological, emotional and behavioral support from the start to adaptation, social reintegration, and developing skills is needed; social groups of patients and supporting persons should be conducted [39].

Powered robotic exoskeletons represent a new technology aimed to improve walking with lower extremity weakness. Early conventional physical therapy of walking after stroke improves speed and endurance, but it is very demanding for the therapists. Exoskeletons reduce the need for physical strain, and also enable repetitions. Treadmill-based robotics was also developed leading to improvement of walking after stroke, but without change in speed and endurance. Current clinical studies explore safety and efficiency of exoskeletons for gait in subacute and chronic stroke, with more convincing results in the sub-acute phase $[40,41]$.

Virtual reality (VR) is a technology generated by the computer with interaction with a user, enabling precise movements using sensorimotor feedback. Recent studies have confirmed effectiveness of VR in stroke rehabilitation, especially for gait and balance. More flexible software and individualized treatment are needed.

VR enables acquisition and retention of motor skills through repetitive, defined, motivating task specific training. Current metacentric studies of VR in stroke rehabilitation are limited, mainly because of study designs and small sample size [42-45].

Noninvasive brain stimulation (NIBS) facilitates neuroplasticity. Transcranial magnet stimulation (TMS) and transcranial direct current stimulation (tDCS) are commonly used for noninvasive brain stimulation. The use of NIBS demands meaningful rehabilitation program that is task specific. It should be applied with other procedures that promote neuroplasticity, such as body weight support treadmill training, functional electric stimulation of the lower limb and bio feedback. Adequate timing of NIBS during a gait cycle is mandatory. Important questions for adequate use of NIBS are: which hemisphere should be stimulated, location and extent of stimulation, and individualization of treatment. Because of high individual variability of patients to NIBS customized stimulation is recommended. Furthermore, genetic and imaging biomarkers to predict outcome, and definition of target sites should be clarified in the future [46].

Functional MRI has confirmed that motor recovery of paretic limb after stroke is associated with hyper activation in different brain areas, such as contralesional hemisphere and subcortical structures. Mental practice is internal stimulation created by auditory, visual, tactile, or kinesthetic stimulation without movement. Mental practice and physical therapy are used in post-stroke rehabilitation [47].

Sleep problems in stroke are frequent and multifactorial, sometimes reducing acute recovery with longer hospitalization, fatigue, demotivation, and sedentary life style. Sleep is also of primary importance for neuroplasticity and memory consolidation. Transcranial direct current stimulation (tDCS) for sleep disorders after stroke is a safe technology that alters brain plasticity, modifies neuronal activity, delivering a weak electric current (1-2 mA). Both anodal and cathodal tDCS are used. One randomized study confirmed that anodal tDCS produced performance gains in the paretic hand. Studies that analyzed motor rehabilitation program combined with tDCS indicate additional improved motor outcomes. Further studies are needed. Individual variations caused by mental health status, motivation, and many physical factors, such as fatigue, significantly influence the outcome of use of tDCS [48-50].

In conclusion, scientific evidence is sufficient to confirm the necessity for physical rehabilitation of patients after stroke. It represents the cornerstone during the early phase and continues throughout the whole post stroke period.

\section{References}

1. de Oliveira Manoel AL, Goffi A, et al. The critical care management of spontaneous intracranial hemorrhage: a contemporary review. Crit Care 2016 18;20:272. doi: 10.1186/ s13054-016-1432-0.

2. Heron N, Kee F, Donnelly M, Cupples ME. Systematic review of rehabilitation programmes initiated within 90 days of a transient ischaemic attack or 'minor' stroke: a protocol. BMJ Open 2015;5(6):e007849. doi: 10.1136/bmjopen-2015-007849
3. Hankey GJ. Stroke. Lancet 2016. pii: S0140-6736(16)30962-X. doi: 10.1016/S0140-6736(16)30962-X.

4. Peisker T, Koznar B, Stetkarova I, Widimsky P. Acute stroke therapy: A review. Trends Cardiovasc Med 2016. pii: S10501738(16)30086-X. doi: 10.1016/j.tcm.2016.06.009.

5. Prabhakaran S, Ruff I, Bernstein RA. Acute stroke intervention: a systematic review. JAMA. 2015; 313(14):1451-1462. doi: 10.1001/jama.2015.3058 
6. Chan B. Effect of increased intensity of physiotherapy on patient outcomes after stroke: an economic literature review and costeffectiveness analysis. Ont Health Technol Assess Ser 2015; 15(7):1-43.

7. Kramer S, Johnson L, Bernhardt J, Cumming T. Energy expenditure and cost during walking after stroke: a systematic review. Arch Phys Med Rehabil 2016; 97(4):619-32. e1. doi: 10.1016/j.apmr.2015.11.007.

8. Kristensen $\mathrm{OH}$, Stenager E, Dalgas U. Muscle strength and poststroke hemiplegia: a systematic review of muscle strength assessment and muscle strength impairment. Arch Phys Med Rehabil 2017; 98(2):368-380. doi: 10.1016/j.apmr.2016.05.023.

9. Pollock A, Farmer SE, Brady MC, Langhorne P, Mead GE, Mehrholz J, van Wijck F. Interventions for improving upper limb function after stroke. Cochrane Database Syst Rev 2014; (11):CD010820. doi: 10.1002/14651858. CD010820.pub2.

10. Elsner B, Kugler J, Pohl M, Mehrholz J. Transcranial direct current stimulation (tDCS) for improving activities of daily living, and physical and cognitive functioning, in people after stroke. Cochrane Database Syst Rev 2016; 3:CD009645. doi: 10.1002/14651858. CD009645.pub3.

11. das Nair $R$, Cogger $H$, Worthington $E$, Lincoln NB. Cognitive rehabilitation for memory deficits after stroke. Cochrane Database Syst Rev 2016; 9:CD002293.

12. Brady MC, Kelly H, Godwin J, Enderby P, Campbell P. Speech and language therapy for aphasia following stroke. Cochrane Database Syst Rev 2016; (6):CD000425. doi: 10.1002/14651858.CD000425.pub4

13. Kessner SS, Bingel U, Thomalla G. Somatosensory deficits after stroke: a scoping review. Top Stroke Rehabil 2016; 23(2):136-146. doi: 10.1080/10749357.2015.1116822.

14. Feigin VL, Norrving B, George MG, Foltz JL, Roth GA, Mensah GA. Prevention of stroke: a strategic global imperative. Nat Rev Neurol 2016; 12(9):501-512. doi: 10.1038/nrneurol.2016.107.

15. Jellema S, van der Sande R, van Hees S, Zajec J, Steultjens EM, Nijhuis-van der Sanden MW. Role of environmental factors on resuming valued activities poststroke: a systematic review of qualitative and quantitative findings. Arch Phys Med Rehabil 2016; 97(6):991-1002.e1. doi: 10.1016/j.apmr.2016.01.015

16. Lawton M, Haddock G, Conroy P, Sage K. Therapeutic alliances in stroke rehabilitation: a meta-ethnography. Arch Phys Med Rehabil 2016; 97(11):1979-1993. doi: 10.1016/j.apmr.2016. 03.031 .

17. Harvey RL. Predictors of functional outcome following stroke. Phys Med Rehabil Clin N Am 2015; 26(4):583-598. doi: 10.1016/ j.pmr.2015.07.002.

18. Mees M, Klein J, Yperzeele L, Vanacker P, Cras P. Predicting discharge destination after stroke: A systematic review. Clin Neurol Neurosurg 2016; 142:15-21. doi: 10.1016/j.clineuro. 2016.01.004.

19. Sauzet O, Kleine M, Menzel-Begemann A, Exner AK. Longitudinal randomised controlled trials in rehabilitation poststroke: a systematic review on the quality of reporting and use of baseline outcome values. BMC Neurol 2015; 15: 99. doi: 10.1186/s12883-015-0344-y.

20. Ehrensberger M, Simpson D, Broderick P, Monaghan K. Crosseducation of strength has a positive impact on post stroke rehabilitation: a systematic literature review. Top Stroke Rehabil 2016; 23(2):126-135. doi: 10.1080/10749357.2015. 1112062

21. Luker J, Lynch E, Bernhardsson S, Bennett L, Bernhardt J. Stroke Survivors' experiences of physical rehabilitation: a systematic review of qualitative studies. Arch Phys Med Rehabil. 2015; 96(9):1698-708.e10. doi: 10.1016/j.apmr.2015.03.017.

22. Stinear CM. Stroke rehabilitation research needs to be different to make a difference. F1000Res 2016; 5. pii: F1000 Faculty Rev1467. doi: 10.12688/f1000research.8722.1.

23. Chen J, Jin W, Zhang XX, Xu W, Liu XN, Ren CC. Telerehabilitation approaches for stroke patients: systematic review and meta-analysis of randomized controlled trials. J Stroke Cerebrovasc Dis 2015; 24(12):2660-2668. doi: 10.1016/ j.jstrokecerebrovasdis. 2015.09.014
24. Parke HL, Epiphaniou E, Pearce G, Taylor SJ, Sheikh A, Griffiths CJ, et al. Self-Management support interventions for stroke survivors: a systematic meta-review. PLoS One 2015;10(7):e0131448. doi: 10.1371/journal.pone.0131448.

25. Clarke DJ, Forster A. Improving post-stroke recovery: the role of the multidisciplinary health care team. J Multidiscip Healthc 2015; 8:433-442. doi: 10.2147/JMDH.S68764.

26. Bank J, Charles K, Morgan P. What is the effect of additional physiotherapy on sitting balance following stroke compared to standard physiotherapy treatment: a systematic review. Top Stroke Rehabil. 2016; 23(1):15-25. doi: 10.1179/1945511915Y. 0000000005 .

27. Sehatzadeh S. effect of increased intensity of physiotherapy on patient outcomes after stroke: an evidence-based analysis. Ont Health Technol Assess Ser 2015; 15(6):1-42

28. Bernhardt J, English C, Johnson L, Cumming TB. Early mobilization after stroke: early adoption but limited evidence. Stroke 2015; 46(4):1141-6. doi: 10.1161/STROKEAHA.114. 007434.

29. Everink IH, van Haastregt JC, van Hoof SJ, Schols JM, Kempen GI. Factors influencing home discharge after inpatient rehabilitation of older patients: a systematicreview. BMC Geriatr 2016; 16:5. doi: 10.1186/s12877-016-0187-4.

30. Pin-Barre C, Laurin J. Physical Exercise as a diagnostic, rehabilitation, and preventive tool: influence on neuroplasticity and motor recovery after stroke. Neural Plast 2015;2015:608581. doi: $10.1155 / 2015 / 608581$

31. Saunders DH, Sanderson M, Hayes S, et al. Physical fitness training for stroke patients. Cochrane Database Syst Rev 2016; 3:CD003316. doi: 10.1002/14651858.CD003316.pub6.

32. Ammann BC, Knols RH, Baschung P, de Bie RA, de Bruin ED. Application of principles of exercise training in sub-acute and chronic stroke survivors: a systematic review. BMC Neurol 2014; 14:167. doi: 10.1186/s12883-014-0167-2

33. Gomes-Neto M, Saquetto MB, Silva CM, Carvalho VO, Ribeiro $\mathrm{N}$, Conceição C. Effects of respiratory muscle training on respiratory function, respiratory muscle strength, and exercise tolerance in patients poststroke: a systematic review with metaanalysis. Arch Phys Med Rehabil. 2016; 97(11):1994-2001. doi:10.1016/j.apmr.2016.04.018.

34. Mohd Zulkifly MF, Ghazali SE, Che Din N, Ajit Singh DK, Subramaniam P. A review of risk factors for cognitive impairment in stroke survivors. ScientificWorldJournal. 2016; 2016:3456943. doi: 10.1155/2016/3456943.

35. Constans A, Pin-Barre C, Temprado JJ, Decherchi P, Laurin J. Influence of aerobic training and combinations of interventions on cognition and neuroplasticity after stroke. Front Aging Neurosci 2016;8:164. doi: 10.3389/fnagi.2016.00164.

36. Tiozzo E, Youbi M, Dave K, Perez-Pinzon M, Rundek T, Sacco RL, et al.Aaerobic, resistance, and cognitive exercise training poststroke. Stroke 2015; 46(7):2012-2016. doi: 10.1161/ STROKEAHA.114.006649.

37. Austin MW, Ploughman M, Glynn L, Corbett D. Aerobic exercise effects on neuroprotection and brain repair following stroke: a systematic reviewand perspective. Neurosci Res 2014; 87:8-15. doi: 10.1016/j.neures.2014.06.007.

38. Kendall BJ, Gothe NP. Effect of aerobic exercise interventions on mobility among stroke patients: a systematic review. Am J Phys Med Rehabil 2016; 95(3):214-224. doi: 10.1097/PHM 0000000000000416

39. Pearce G, Pinnock H, Epiphaniou E, Parke HL, Heavey E, Griffiths CJ, et al. Experiences of self-management support following a stroke: a meta-review of qualitative systematic reviews. PLoS One 2015; 10(12):e0141803. doi: 10.1371/ journal.pone.0141803

40. Louie DR, Eng JJ. Powered robotic exoskeletons in poststroke rehabilitation of gait: a scoping review. J Neuroeng Rehabil 2016; 13(1):53. doi: 10.1186/s12984-016-0162-5.

41. Veerbeek JM, Langbroek-Amersfoort AC, van Wegen EE, Meskers CG, Kwakkel G. Effects of robot-assisted therapy for the upper limb after stroke: a systematic review and meta-analysis. Neurorehabil Neural Repair 2017; 31(2):107-121. doi: 10.1177/ 1545968316666957. 
42. Luque-Moreno C, Ferragut-Garcías A, Rodríguez-Blanco C, Heredia-Rizo AM1, Oliva-Pascual-Vaca J, Kiper P, OlivaPascual-Vaca Á. A decade of progress using virtual reality for poststroke lower extremity rehabilitation: systematic review of the intervention methods. Biomed Res Int 2015;2015:342529. doi: $10.1155 / 2015 / 342529$

43. Teo WP, Muthalib M, Yamin S, Hendy AM, Bramstedt K, Kotsopoulos E, Perrey S, Ayaz H. Does a combination of virtual reality, neuromodulation and neuroimaging provide a comprehensive platform for neurorehabilitation? - A narrative review of the literature. Front Hum Neurosci 2016; 10:284. doi: 10.3389/fnhum.2016.00284.

44. Laver K, George S, Thomas S, Deutsch JE, Crotty M. Virtual reality for stroke rehabilitation: an abridged version of a Cochrane review. Eur J Phys Rehabil Med 2015; 51(4):497-506.

45. Darekar A, McFadyen BJ, Lamontagne A, Fung J. Efficacy of virtual reality-based intervention on balance and mobility disorders post-stroke: a scoping review. J Neuroeng Rehabil 2015; 12:46. doi: 10.1186/s12984-015-0035-3.
46. Xu Y, Hou QH, Russell SD, Bennett BC, Sellers AJ, Lin Q, Huang DF. Neuroplasticity in post-stroke gait recovery and noninvasive brain stimulation. Neural Regen Res 2015; 10(12): 2072-2080. doi: 10.4103/1673-5374.172329.

47. Peters HT, Edwards DJ, Wortman-Jutt S, Page SJ. Moving forward by stimulating the brain: transcranial direct current stimulation in post-strokehemiparesis. Front Hum Neurosci 2016; 10:394. doi: 10.3389/fnhum.2016.00394.

48. Ebajemito JK, Furlan L, Nissen C, Sterr A. application of transcranial direct current stimulation in neurorehabilitation: the modulatory effect of sleep. Front Neurol 2016;7:54. doi: 10.3389/fneur.2016.00054

49. Smith MC, Stinear CM. Transcranial magnetic stimulation (TMS) in stroke: Ready for clinical practice? J Clin Neurosci 2016;31:10-14. doi: 10.1016/j.jocn.2016.01.034.

50. Peters HT, Page SJ. integrating mental practice with task-specific training and behavioral supports in poststroke rehabilitation: evidence, components, and augmentative opportunities. Phys Med Rehabil Clin N Am 2015; 26(4):715-727. doi: 10.1016/j.pmr. 2015.06.004 